\title{
Screening wells by multi-scale grids for multi-stage Markov Chain Monte Carlo simulation
}

\section{Akbari, Hani; Engsig-Karup, Allan Peter}

Published in:

Mathematics and Computers in Simulation

Link to article, DOI:

10.1016/j.matcom.2018.03.014

Publication date:

2018

Document Version

Peer reviewed version

Link back to DTU Orbit

Citation (APA):

Akbari, H., \& Engsig-Karup, A. P. (2018). Screening wells by multi-scale grids for multi-stage Markov Chain Monte Carlo simulation. Mathematics and Computers in Simulation, 151, 15-28.

https://doi.org/10.1016/j.matcom.2018.03.014

\section{General rights}

Copyright and moral rights for the publications made accessible in the public portal are retained by the authors and/or other copyright owners and it is a condition of accessing publications that users recognise and abide by the legal requirements associated with these rights.

- Users may download and print one copy of any publication from the public portal for the purpose of private study or research.

- You may not further distribute the material or use it for any profit-making activity or commercial gain

- You may freely distribute the URL identifying the publication in the public portal 


\section{Accepted Manuscript}

Screening wells by multi-scale grids for multi-stage Markov Chain Monte Carlo simulation

Hani Akbari, Allan P. Engsig-Karup

PII: $\quad$ S0378-4754(18)30086-7

DOI: $\quad$ https://doi.org/10.1016/j.matcom.2018.03.014

Reference: $\quad$ MATCOM 4561

To appear in: Mathematics and Computers in Simulation

Received date: 25 February 2017

Revised date: 18 February 2018

Accepted date : 26 March 2018

Please cite this article as: H. Akbari, A.P. Engsig-Karup, Screening wells by multi-scale grids for multi-stage Markov Chain Monte Carlo simulation, Math. Comput. Simulation (2018), https://doi.org/10.1016/j.matcom.2018.03.014

This is a PDF file of an unedited manuscript that has been accepted for publication. As a service to our customers we are providing this early version of the manuscript. The manuscript will undergo copyediting, typesetting, and review of the resulting proof before it is published in its final form. Please note that during the production process errors may be discovered which could affect the content, and all legal disclaimers that apply to the journal pertain. 


\title{
Screening wells by multi-scale grids for multi-stage Markov Chain Monte Carlo simulation
}

\author{
Hani Akbari ${ }^{a, b, *}$, Allan P. Engsig-Karup ${ }^{a, b}$ \\ ${ }^{a}$ Department of Applied Mathematics and Computer Science, Technical University of Denmark \\ ${ }^{b}$ Center for Energy Resources Engineering (CERE), Technical University of Denmark
}

\begin{abstract}
For improved prediction of subsurface flows and representation of the uncertainties of geostatistical properties, we use the framework of Bayesian statistical interface in combination with the Markov Chain Monte Carlo (MCMC) method which needs many fine-scale simulations. Hence it is essential to apply cheap screening stages, such as coarse-scale simulation to remove irrelevant proposals of the generated Morkov chain, reduce fine-scale computational cost and increase the acceptance rate of MCMC. We propose a screening step, that is examination of subsurface characteristics around injection/production wells, aiming at accurate breakthrough capturing as well as above mentioned efficiency goals. However this short time simulation needs fine-scale structure of the geological model around wells and running a fine-scale model is not as cheap as necessary for screening steps. On the other hand applying it on a coarse-scale model declines important data around wells and causes inaccurate results, particularly accurate breakthrough capturing which is important for prediction applications. Therefore we propose a multi-scale grid which preserves the fine-scale model around wells (as well as high permeable regions and fractures) and coarsens rest of the field and keeps efficiency and accuracy for the screening well stage and coarse-scale simulation, as well. A discrete wavelet transform is used as a powerful tool to generate the desired unstructured multi-scale grid efficiently. Finally an accepted proposal on coarse-scale models (screening well stage and coarse-scale simulation) will be assessed by fine-scale simulation. Accepted proposals are saved for prediction. Numerical results admit increment in acceptance rate, improvement in breakthrough capturing and significant reduction in computational cost by avoiding many forward simulations.
\end{abstract}

Keywords: Uncertainty quantification, multi-stage Markov Chain Monte Carlo simulation, discrete wavelets transform, screening wells, multi-scale grid, reservoir simulation.

\section{Introduction}

Reliable characterization of subsurface is an important part of accurately predicting reservoir behaviour and flow patterns which are of high interest in oil and gas recovery or $\mathrm{CO}_{2}$ sequestration. Uncertainties of porosity and permeability for a large number of grid cells, becomes a critical challenge in subsurface characterization, since very little data (such as phase fractional flow or transient pressure (dynamic data) and porosity and permeability at injection/production wells (static data)) is available for subsurface characterization. Reducing and quantifying these uncertainties can be achieved by comparison between available dynamic data with response of the physical model of the reservoir to a chain of

\footnotetext{
This research project is financially supported by The Danish Advanced Technology Foundation in the OPTION Project (J.nr 6320133).

* Corresponding author

Email addresses: haniakbari@gmail.com (Hani Akbari), apek@dtu.dk (Allan P. Engsig-Karup)
} 
proposed geological models (realizations) including static data. Hence we face an inverse problem aiming at reconstructing the spatial distribution of the permeability field as the main parameter of the geological model.

However nonlinear properties of flow and transport in porous media flows obstruct direct calculation of the probability distribution for permeability field conditioned to dynamic data. Instead we can estimate the probability distribution which are the outcomes of a large number of realizations. Moreover such estimation can be valid if the permeability realizations correctly reflect the uncertainty of subsurface characteristics. Hence it is essential to consider a reasonable prior covariance of permeability field and it is then possible to specify full prior distribution. In brief assuming known prior distribution, we generate large number of realizations and calculate the response or outcome of each realization (forward simulation) to estimate probability distribution of the permeability field. Such a probability distribution can be used in evaluation of screening criteria for well performances.

Markov Chain Monte Carlo (MCMC) method is a common choice to generate a reliable chain of proposals that needs forward simulation for each proposal and then decides to accept or reject that proposal. However, MCMC is very expensive in computational cost and accepts only a small portion of proposals since it runs many forward simulations also for the irrelevant proposals that should be rejected immediately. Hence it is essential to reject an irrelevant proposal by cheap screening steps (before forward simulation) to reduce computational cost and increase the acceptance rate.

Several developments for efficient MCMC have been proposed; for example employing a perturbation technique to identify irrelevant proposals [15], conditioning to the pressure data [14], blocking MCMC [13] and running MCMC on different scales [10]. A two-stage MCMC is proposed in [16, 17] that employs coarse scale model based on single-phase coarsening of the permeability field. At first screening step a proposal is assessed with coarse-scale simulation and if it passed the screening test then a forward simulation will be run to accept or reject it. In [18] a multi-stage MCMC including a tracer test as a screening step before coarse-scale simulation has been developed.

We propose a new screening step which evaluates the response of a proposal (phase fractional curve) around wells. The practical issues and engineering aspects of this approach are addressed in [19, 20]. Within short time of a wetting phase injection (for example super critical $\mathrm{CO}_{2}$ or water in two-phase models) in both injection and production wells, we can obtain phase fractional curves to assess a proposal. More precisely we follow these steps:

1. For a short time interval, inject in injection wells and produce in production wells. The updated state of the reservoir would be the initial state of the reservoir for next step.

2. Having a initial state of the reservoir, inject in production wells and produce in injection wells then gives phase fractional curves corresponding to the injection wells. The updated state of the reservoir would be the initial state of the reservoir for next step.

3. From the latest state of the reservoir, again inject in injection wells and produce in production wells which gives phase fractional curves corresponding to the production wells.

4. Use the estimated phase fractional curves to assess the proposal.

Since in the screening wells stage we evaluate features of the permeability field around wells, it is essential to preserve fine-scale structure of the permeability field around wells, otherwise applying this approach on coarse-scale model declines important data around wells that causes inaccurate evaluation, in particular long delay in breakthrough regarding to dissipative flow behaviour of coarsened blocks. If we run a simulation on fine-scale, even for a short time in comparison with full simulation time, this approach might be computationally intractable. Hence we need a multi-scale grid which preserves finescale features around wells as well as high permeable regions and fractures, and coarsens the rest of the field. This intelligent grid keeps efficiency and accuracy for screening well step and coarse-scale simulation. In $[11,12,7]$ a discrete wavelet transform is employed to generate such a coarse-scale grid for two and three dimensional reservoirs and have shown superiority of this model over conventional 
coarsening methods. We follow their approach to generate our coarse-scale model, both for the screening wells stage and coarse-scale simulation.

The organization of the paper is as follows. In next section we introduce a two-phase (oil and water) model and governing equations. Then we give a brief explanation about generating a coarse-scale model by a discrete wavelet transform. In section 3, the multi-stage MCMC and screening wells will be described. In section 4, we employ a Karhunen-Loeve expansion to obtain prior distribution and represent a random permeability field. Finally numerical results to show improvement in acceptance rate are presented in section 5 .

\section{Governing Equations and Coarse Scale Model}

Governing Equations. A full description of subsurface flow in porous media together with numerical techniques of discretization and solution can be found in literature [8,9]. The mass conservation law for incompressible, immiscible two-phase (oil and water) flow can be stated as

$$
\phi \frac{\partial S_{\alpha}}{\partial t}+\nabla \cdot \mathbf{u}_{\alpha}=q_{\alpha}, \quad q_{\alpha}=\frac{\tilde{q}_{\alpha}}{\rho_{\alpha}}, \quad \alpha=o, w
$$

where $\phi, S_{\alpha}, \rho_{\alpha}, \tilde{q}_{\alpha}$ and $u_{\alpha}$ denote respectively, porosity, saturation, density, source/sink term and Darcy's velocity for phase $\alpha=o, w$. For simplicity, but without loss of generality, we choose to ignore gravity and capillary effects in the formulation. Darcy's velocity can then be written as:

$$
\mathbf{u}_{\alpha}=-\kappa \lambda_{\alpha} \nabla p
$$

where $\kappa, \lambda_{\alpha}$ and $p$ denote the absolute permeability field, phase mobility and pressure, respectively. Hence we have a system of two equations in the form:

$$
\phi \frac{\partial S_{\alpha}}{\partial t}+\nabla \cdot\left(-\kappa \lambda_{\alpha} \nabla p\right)=q_{\alpha}, \quad \alpha=o, w
$$

Summing the two equations of (1) and noting $S_{o}+S_{w}=1$, we obtain

$$
\nabla \cdot \mathbf{u}=q, \quad \mathbf{u}=-\kappa \lambda \nabla p
$$

where $\mathbf{u}=\mathbf{u}_{o}+\mathbf{u}_{w}$ and $\lambda=\lambda_{o}+\lambda_{w}$ are total (Darcy's) velocity and total mobility, respectively, and $q=q_{o}+q_{w}$. The fractional flow of water is defined by $f_{w}=\lambda_{w} / \lambda$, so $\mathbf{u}_{w}=f_{w} \mathbf{u}$ and equation (1) for water phase becomes

$$
\phi \frac{\partial S_{w}}{\partial t}+\nabla \cdot\left(f_{w} \mathbf{u}\right)=q_{w}
$$

By outcome or response of the physical model (of the reservoir) to a proposed geological model (here permeability field), $\kappa$, we mean solving equations (2) and (3) and obtaining phase fractional curve $f_{w}\left(S_{w}(t)\right)$. The first equation is an elliptic equation called pressure equation while the second one is a conservation law called transport equation and has a hyperbolic nature. We solve them sequentially as a decoupled system.

Coarse Scale Model. Coarsening techniques have been largely exploited in literature for reservoir simulation $[23,22]$. In this study we focus on $2 \mathrm{D}$ problems as a proof-of-concept of the proposed MCMC approach for efficient screening of wells, so we introduce the theory in two dimensions that could be extended to three space dimension with no conceptual changes.

Let $\kappa(\mathbf{x})$, with $\mathbf{x} \in \mathbf{\Omega}$, denote the absolute permeability field function that we want to coarsen by means of the discrete wavelet transforms (DWT). To apply DWT, we need a set of functions, called wavelet family, which is constructed by dilation and translation of two main functions $\varphi$, scaling function 

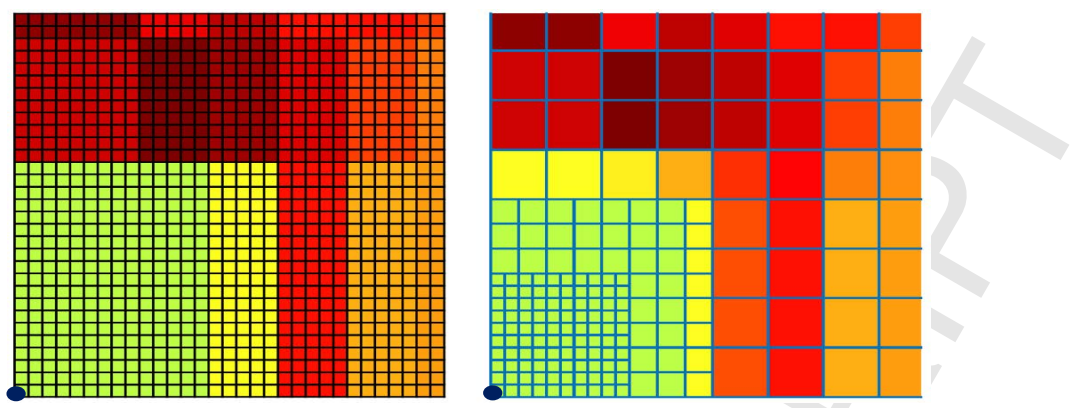

Figure 1: Black circle denotes well location. (Left) Fine scale structure of the permeability field. (Right) Coarse scale model adapted to well, after two level of upscaling that preservers fine-scale structure around well and coarsens rest of the field.

and $\psi$, wavelet function, $[4,3] .\{\varphi, \psi\}$ have two useful properties: they are compactly supported and recursive. The former means that they can be nonzero only in a compact set, the latter that they can be written as a linear combination of their dilation and translation:

$$
\varphi(x)=\sum_{k=k_{0}}^{k_{1}} a_{k} \varphi(2 x-k), \quad \psi(x)=\sum_{k=k_{0}}^{k_{1}} b_{k} \psi(2 x-k),
$$

where $a_{k}$ and $b_{k}$ are known values. These properties make their definite integrals easy to evaluate, and they are highly demanded in the field of data compression and numerical computation, particularly for numerical solution of partial differential equations [6]. Example of data compression can be found in seismic signals which may be acoustics, geological, x-rays or tomographic images, biomedical signals, ultra wide-band wireless communication, etc [5]. Applying DWT on the permeability field translates into the evaluation four types of definite integrals:

$$
\begin{aligned}
S_{i j}=\int_{\Omega} \phi_{i}(x) \phi_{j}(y) \kappa(\mathbf{x}) d \mathbf{x}, & D_{i j}^{h}=\int_{\Omega} \phi_{i}(x) \psi_{j}(y) \kappa(\mathbf{x}) d \mathbf{x}, \\
D_{i j}^{v}=\int_{\Omega} \psi_{i}(x) \phi_{j}(y) \kappa(\mathbf{x}) d \mathbf{x}, & D_{i j}^{d}=\int_{\Omega} \psi_{i}(x) \psi_{j}(y) \kappa(\mathbf{x}) d \mathbf{x},
\end{aligned}
$$

where $\mathbf{x}=(x, y), d \mathbf{x}=d x d y, \varphi_{i}(x)=\varphi(2 x-i)$ and $\psi_{i}(x)=\psi(2 x-i)$. The indices $(i, j)$ indicate a 2 by 2 block of fine-scale cells of the permeability field and $S_{i j}$, called scaling coefficient, is a measure of the content of this block. Large values of the scaling coefficient correspond to a highly permeable block. $D_{i, j}^{l}, l=h, v, d$, called detailed coefficient, expresses the difference between a block and its neighbors in the horizontal, vertical and diagonal direction, respectively. Hence, small value of $D_{i, j}^{l}$ tell us that there are not sharp variations in the permeability field around the block (low contrast block), and if also $S_{i j}$ is small enough (low permeable block), then we will consider it as a one new coarsened cell, otherwise that block is kept intact as it is. This is the first level of upscaling and we may iterate this procedure to obtain the second level of upscaling, where a coarsened cell of level 2 consists in a 4 by 4 block of fine cells. Eventually, the procedure generates a non-uniform mesh because it prevents the agglomeration of cells with highly variable permeability values.

For example the structure of the upscaled mesh around a well is plotted in Figure 1. Considering a well at Down-Left corner, we see around the well, the fine scale of permeability field is retained while far from it coarsened cells of level 1 or 2 are obtained.

After finding the desired coarse grid, we need to solve subsurface equations (2) and (3) over it. To solve the elliptic equation we use a classical finite volume technique, which gives locally conservative fluxes. To solve transport equation we use forward difference time discretization and upwinding. Details 
of the approach can be found in $[12,11]$ for two and three dimensional reservoirs. Now we have a multiscale coarse grid and can solve screening steps over it with reduced computational cost and with desired accuracy. In next section we describe how to generate a chain of permeability fields.

\section{Markov Chain Monte Carlo Method}

Following [16], we use a Bayesian framework to sample permeability field from the conditional distribution $P\left(\kappa \mid F_{r}\right)$. Here $F_{r}$, represents observed (reference) production data and $\kappa$ represents a random permeability field generated by a prior distribution, $P(\kappa)$ that we explain in next section. Using Bayes' theorem we can write

$$
P\left(\kappa \mid F_{r}\right) \propto P\left(F_{r} \mid \kappa\right) P(\kappa),
$$

where $P\left(F_{r} \mid \kappa\right)$ represents likelihood function and requires $F_{\kappa}$, response of the physical model to proposed permeability field, $\kappa$. Hence each likelihood evaluation needs one forward simulation, demanding high computational cost.

We assume the likelihood follows a Gaussian distribution,

$$
P\left(F_{r} \mid \kappa\right) \propto \exp \left(-\frac{1}{2 \sigma^{2}}\left\|F_{\kappa}-F_{r}\right\|^{2}\right)
$$

where $\sigma^{2}$ represents the precision associated with the reference data $F_{r}$ and response $F_{\kappa}$. Gaussian nature of (5), predicts that for accepted proposals, $\left\|F_{\kappa}-F_{r}\right\|^{2}$ converges to (and oscillates around) $\sigma^{2}$ and in this case chain has reached the steady state. In numerical results, we plot shifted logarithmic scale of the error,

$$
E_{\kappa}=\log \left(\left\|F_{\kappa}-F_{r}\right\|^{2}\right)-\log \left(\sigma^{2}\right),
$$

and expect it converges to (and oscillates around) zero. Combining (4) and (5), we denote posterior distribution as

$$
\pi(\kappa):=P\left(\kappa \mid F_{r}\right) \propto \exp \left(-\frac{1}{2 \sigma^{2}}\left\|F_{\kappa}-F_{r}\right\|^{2}\right) P(\kappa),
$$

and sample from the distribution $\pi(\kappa)$ with Metropolis-Hasting MCMC method [2, 1]. We also need a probability distribution, $q(x \mid y)$, which is easy to sample and has an explicit form. Usually a symmetric distribution, $q(x \mid y)=q(y \mid x)$, such as a uniform distribution centered at $y$ is chosen. Metropolis-Hasting MCMC algorithm is as follows:

Algorithm 1. Single-stage MCMC

- Set arbitrary initial permeability field $\kappa_{0}$.

- For $j=1,2,3, \cdots$

1. At previous accepted state $\kappa_{j-1}$, generate $\kappa$ from $q\left(\kappa \mid \kappa_{j-1}\right)$.

2. Accept $\kappa$ with probability $\alpha$, where

$$
\alpha\left(\kappa, \kappa_{j-1}\right)=\min \left(1, \frac{q\left(\kappa_{j-1} \mid \kappa\right) \pi(\kappa)}{q\left(\kappa \mid \kappa_{j-1}\right) \pi\left(\kappa_{j-1}\right)}\right),
$$

i.e. set $\kappa_{j}=\kappa$ with probability $\alpha$ and $\kappa_{j}=\kappa_{j-1}$ with probability $1-\alpha$.

\section{End for}


As we mentioned, a forward simulation is needed to calculate $\pi(\kappa)$ and it would be computationally very expensive for a large number of proposed permeability fields. To alleviate computational cost a few number of cheap screening stages will screen a proposal to determine if forward simulation must also reject it or not. Therefore many irrelevant proposals will be rejected before forward simulation, yielding reduction in computation and improvement in acceptance rate.

Assuming $N_{s}$ screening stages, each stage has its own reference data and likelihood function. Denoting $\left\{F_{r}^{i}\right\}_{i=1}^{N_{s}}$ as reference data of the $i^{t h}$ stage, we run screening simulation to compute the response of screening stage, $F_{\kappa}^{i}$, and then calculate corresponding posterior distribution:

$$
\pi^{i}(\kappa)=P\left(\kappa \mid F_{r}^{i}\right) \propto \exp \left(-\frac{1}{2 \sigma_{i}^{2}}\left\|F_{\kappa}^{i}-F_{r}^{i}\right\|^{2}\right) P(\kappa)
$$

where $\sigma_{i}^{2}$ must be selected carefully. In numerical results we report

$$
\tilde{\sigma}_{i}^{2}=\frac{1}{2 \sigma_{i}^{2}}, \quad i=f, c, w,
$$

where $f, c$ and $w$ are corresponding to forward simulation, coarse scale screening stage and screening well stage, respectively. Multi-stage Metropolis-Hasting MCMC algorithm is as follows:

Algorithm 2. Multi-stage MCMC

(0) Set arbitrary initial permeability field $\kappa_{0}$.

(1) For $j=1,2,3, \cdots$

1. At previous accepted state $\kappa_{j-1}$, generate $\kappa$ from $q\left(\kappa \mid \kappa_{j-1}\right)$.

2. Set $Q^{0}\left(\kappa \mid \kappa_{j-1}\right)=q\left(\kappa \mid \kappa_{j-1}\right)$.

- For $i=1, \cdots, N_{s}$

(a) Run $i^{\text {th }}$ screening simulation to calculate $\pi^{i}(\kappa)$

(b) Accept $\kappa$ with probability $\alpha^{i}$, where

$$
\alpha^{i}\left(\kappa, \kappa_{j-1}\right)=\min \left(1, \frac{Q^{i-1}\left(\kappa_{j-1} \mid \kappa\right) \pi^{i}(\kappa)}{Q^{i-1}\left(\kappa \mid \kappa_{j-1}\right) \pi^{i}\left(\kappa_{j-1}\right)}\right),
$$

(c) If $\kappa$ rejected, set $\kappa_{j}=\kappa_{j-1}$ and go to (1) to generate a new proposal.

End for i.

3. Proposed $\kappa$ passed screening stages. Run forward simulation to compute $\pi(\kappa)$.

4. Accept $\kappa$ with probability $\alpha$, where

$$
\alpha\left(\kappa, \kappa_{j-1}\right)=\min \left(1, \frac{Q^{N_{s}}\left(\kappa_{j-1} \mid \kappa\right) \pi(\kappa)}{Q^{N_{s}}\left(\kappa \mid \kappa_{j-1}\right) \pi\left(\kappa_{j-1}\right)}\right),
$$

i.e. set $\kappa_{j}=\kappa$ with probability $\alpha$ and $\kappa_{j}=\kappa_{j-1}$ with probability $1-\alpha$.

\section{End for j.}

The transition distribution $Q^{i}$ in the algorithm, simply indicates that the chain moves form $\kappa_{j-1}$ to $\kappa$ with probability $\alpha^{i}\left(\kappa, \kappa_{j-1}\right) Q^{i-1}$. Actually it is shown that [16],

$$
\frac{Q^{i}\left(\kappa_{j-1} \mid \kappa\right)}{Q^{i}\left(\kappa \mid \kappa_{j-1}\right)}=\frac{\pi^{i}\left(\kappa_{j-1}\right)}{\pi^{i}(\kappa)}
$$


and for example (10) converts to

$$
\alpha^{i}\left(\kappa, \kappa_{j-1}\right)=\min \left(1, \frac{\pi^{i-1}\left(\kappa_{j-1}\right) \pi^{i}(\kappa)}{\pi^{i-1}(\kappa) \pi^{i}\left(\kappa_{j-1}\right)}\right) .
$$

In this study we use two screening stages, screening wells and coarse scale simulations. In the numerical results we report selected values for $\sigma_{i}^{2}$ in likelihood evaluation (8) and show improvement in acceptance rate and efficiency, but before that we explain prior distribution $P(\kappa)$ in $(4)$ or $(8)$.

\section{Prior Distribution}

As the uncertainty space describing the permeability field can be exceptionally large, a reduction of the space dimension must be performed for computationally feasible simulations. The Karhunen-Loeve (KL) expansion [21], allows for the desired reduction that relies on a basic prior information on the structure of the permeability field. In practice very limited information is available to use. We follow $[16,18]$ to apply this reduction.

A standard assumption in geostatistics is to model the permeability to follow a log-normal distribution, i.e., $\log [\kappa(\mathbf{x}, \omega)]=Y(\mathbf{x}, \omega)$, where $\mathbf{x}=(x, y) \in \Omega \in R^{2}$, and $\omega$ is a random element in a probability space. $Y(x, \omega)$ is a field possessing a Gaussian distribution and a covariance function such as

$$
R\left(\mathbf{x}_{1}, \mathbf{x}_{2}\right)=\sigma_{G}^{2}\left(-\frac{\left|x_{1}-x_{2}\right|^{2}}{2 L_{x}^{2}}-\frac{\left|y_{1}-y_{2}\right|^{2}}{2 L_{y}^{2}}\right),
$$

where $L_{x}$ and $L_{y}$ are correlation lengths in $x$ - and $y$-direction, respectively, and $\sigma_{Y}^{2}=E\left[Y^{2}\right]$.

We now briefly describe the essentials of the KL expansion. Suppose $Y(\mathbf{x}, \omega)$ is a second-order stochastic process, that is, $Y(\mathbf{x}, \omega) \in L^{2}(\Omega)$ with a probability of one. We will assume that $E[Y(\mathbf{x}, \omega)]=0$. Given an arbitrary orthonormal basis $\left\{\phi_{i}\right\}$ in $L^{2}$, we can expand $Y(\mathbf{x}, \omega)$ as

$$
Y(\mathbf{x}, \omega)=\sum_{i=1}^{\infty} Y_{i}(\omega) \phi_{i}(\mathbf{x}), \quad \text { with } \quad Y_{i}(\omega)=\int_{\Omega} Y(\mathbf{x}, \omega) \phi_{i}(\mathbf{x}) d \mathbf{x}
$$

functions of the random variable. $\phi_{i}$ is an eigen-function of integral equation involving $R\left(\mathbf{x}_{1}, \mathbf{x}_{2}\right)$ expressed as

$$
\int_{\Omega} R\left(\mathbf{x}_{1}, \mathbf{x}_{2}\right) \phi_{i}\left(\mathbf{x}_{2}\right) d \mathbf{x}_{2}=\lambda_{i} \phi_{i}\left(\mathbf{x}_{1}\right), \quad i=1,2,3, \ldots
$$

where $\lambda_{i}=E\left[Y_{i}^{2}\right]>0$. Denoting $\theta_{i}=Y_{i} / \sqrt{\lambda_{i}}$ then $\theta_{i}$ satisfies $E\left(\theta_{i}\right)=0$ and

$$
Y(\mathbf{x}, \omega)=\sum_{i=1}^{\infty} \sqrt{\lambda_{i}} \theta_{i}(\omega) \phi_{i}(\mathbf{x})
$$

where $\phi_{i}$ and $\lambda_{i}$ satisfy (12). We assume that eigenvalues are ordered so that $\lambda_{1} \geq \lambda_{2} \geq \ldots$

The expansion (13) is called the Karhunen-Loeve expansion. In (12), the $L^{2}$ basis functions $\phi_{i}(\mathbf{x})$ are deterministic and resolve the spatial dependence of the permeability field. The uncertainty is represented by the scalar standard normal random variables $\theta_{i}$.

In general, we only need to keep the leading order terms (quantified by the magnitude of $\lambda_{i}$ ) and still capture most of the energy of the stochastic process $Y(\mathbf{x}, \omega)$. For a truncated $N$-term KL expansion (13) becomes

$$
Y_{N}=\sum_{i=1}^{N} \sqrt{\lambda_{i}} \theta_{i} \phi_{i}
$$

If $\lambda_{i}$ decays very fast, then the truncated KL expansion (14) is a good approximation of the stochastic process in the $L^{2}$ sense. Therefore we sample for random variables $\theta_{i}$, calculate the truncated KL expansion (14) and derive proposed permeability field $\kappa=\exp \left(Y_{N}\right)$. 


\section{Numerical Results}

We consider two test cases to examine the effect of a screening well stage in multi-stage MCMC simulation. For each test case, we consider a square grid and set an injection well at down-left corner and a production well at up-right corner. Considering two-phase oil and water model, we inject water to obtain the oil fractional curve as dynamic data to evaluate likelihood function. After finishing MCMC simulations and deriving some permeability fields for prediction study, we continue injecting water to obtain fractional curve for longer time. The vertical lines in Figures 8 and 15 separate time interval for MCMC simulations and predicting study. Chosen values for parameters are reported in Table 1. We follow these steps for comparison study:

1. With chosen values for correlation lengths $L_{x}, L_{y}$ and variance $\sigma_{G}^{2}$ in covariance function (11), we calculate eigen-pairs $\left(\lambda_{i}, \phi_{i}\right)$ for (12) and plot some of the dominant eigenvalues to see they decay very fast. Reference permeability field is a random linear combination of almost all eigenvectors.

2. On reference permeability field we run forward simulation, coarse-scale simulation and screening well simulation to obtain reference dynamic data for likelihood function and predicting study as well.

3. We select a few number of dominant eigenvalues, $N_{e v}$ in Table 1 , such that sum of them is almost $95 \%$ of sum of all eigenvalues yielding $5 \%$ error if we truncate (13) after $N_{e v}$ term. Therefore with only $N_{e v}$ random numbers, we generate a fine-scale permeability field which is a significant dimension reduction.

4. For probability distribution $q(x \mid y)$ in Algorithms 1 and 2, we use $q(x \mid y)=y+\delta_{q} \epsilon$, where $\epsilon$ is a standard normal distribution.

Now we can run MCMC iterations with or without screening stages. We compare three types of MCMC simulations:

1. Single-stage MCMC. It is presented in Algorithm 1 and no screening stage is considered, hence expensive computation with low acceptance rate is expected.

2. Two-stage MCMC. In Algorithm 2, we consider only coarse-scale simulation to screen proposals before forward simulation. Hence many irrelevant proposals will be rejected by a cheap screening step. Consequently we expect to assess much more proposals and improve acceptance rate, while decreasing computational cost.

3. Three-stage MCMC. In Algorithm 2, we consider two screening stages, at first screening wells stage and then coarse scale simulation, before forward simulation. Since screening well stage runs on coarse scale model, as well as coarse-scale simulation, we do not expect much reduction in computational cost in comparison with two-stage MCMC, but expect to improve acceptance rate significantly.

Case 1. Referring to Table 1, we construct a reference permeability field of dimension $64 \times 64$, and with $N_{e v}=30$ random values we generate proposed permeability fields. Fast decaying of eigenvalues in KL expansion (14) is plotted in Figure 2.

We run three types of MCMC simulations until we obtain 400 accepted proposals. Shifted errors for accepted proposals by forward simulation, equation (6), are plotted in Figure 3. We see shifted errors converge to zero after obtaining a few accepted proposals and oscillate there afterwards, indicating chain is converged to steady state. Therefore we can expect nearly fixed acceptance rate as plotted in Figure 4. Number of evaluated proposals and accepted by each stage are reported in Table 2. For example in three-stage MCMC, 1250 forward simulation is needed to obtain 400 accepted proposals, while 11600 permeability fields were proposed and most of them were rejected by screening stages.

We can take a closer look at three-stage MCMC by plotting shifted errors for accepted proposals by each screening stage and forward simulation. Figure 5 indicates convergence of each stage and a nearly fixed acceptance rate is expected for each stage as we can see in Figure 6. 
To study the predictions, we consider the average of five accepted permeability fields (of last 100 accepted proposals) and plot them in Figure 7. We run fine-scale simulation on each field to produce the rest of the oil fractional curve and compare with reference field. Results are plotted in Figure 8, indicating quality of screening stages to capture the observed dynamic data correctly.

Case 2. In this example we consider a different permeability field (and therefore different fractional curve and breakthrough) and repeat approach of Case 1 to examine acceptance rate of three-stage MCMC and capturing dynamic data. Referring to Table 1, reference permeability field has dimension of $72 \times 72$. Regarding to Figure $9, N_{e v}=40$ random values are enough to generate proposals.

We run three types of MCMC simulations until we obtain 180 accepted proposals. Shifted errors and acceptance rate are plotted in Figures 10 and 11, respectively. Number of evaluated proposals and accepted by each stage are reported in Table 3. Moreover for three-stage MCMC and in Figures 12 and 13, we plotted shifted errors and acceptance rate, respectively.

Finally for predicting study, we consider average of five accepted permeability fields (of last 50 accepted proposals) and plot them in Figure 14. Comparison results of oil fractional curves are plotted in Figure 8 , and again it indicates quality of screening stages to capture the observed dynamic data correctly, in particular breakthrough capturing which is enlarged for comparison.

Addressing CPU-time comparison is highly case dependent. For example, assume that $N_{a}$ simulations are needed for each stage of a multi-stage MCMC. a refers to forward simulation $(f)$, coarse scale simulation $(c)$ and well screening simulation $(w)$. Also assume that $T_{a}, a=f, c, w$, denotes the CPU-time of the correspond simulation. For example the total CPU-time of a three-stage MCMC can then be estimated as $N_{f} T_{f}+N_{c} T_{c}+N_{w} T_{w}$. To address the speed up, the critical question would be the ratio $T_{f} / T_{c}$, or how much a coarse scale simulation is faster than a forward simulation. For example in [12] for some test cases more than 200 times speed-up is reported. In [7], at least 25 times speed-up is obtained for a relatively large-scale problem. We are not worried about $T_{w}$, since it always equals a small portion of $T_{c}$; Actually well screening simulation is a (modified) coarse scale simulation with a shorter time of simulation. We did not give speedup measures in this paper, since it is a proof of concept on the algorithmic approach for well screening.

\section{Conclusion}

To improve efficiency of MCMC simulation i.e. more accurately capturing the dynamic data and removing irrelevant proposals before expensive forward simulation step, we developed a screening well stage on multi-scale and unstructured coarse scale model obtained by discrete wavelet transform of main grid. Incorporating this stage in multi-stage MCMC, we demonstrated that we could use much fewer forward simulations, in comparison with single stage and two stage MCMC. Hence we could improve acceptance rate and reduce computational cost considerably. Also a study of the predictions confirmed that results of multi-stage MCMC can predict reservoir behaviour correctly.

\section{Acknowledgements}

Hani Akbari thanks Professors Luis Felipe Pereira at the University of Texas at Dallas and Victor Ginting at the University of Wyoming for their useful consultations and suggestions. This research project is financially supported by The Danish Advanced Technology Foundation in the OPTION Project (J.nr $6320133)$.

\section{References}

[1] C. Robert and G. Casella, Monte Carlo Statistical Methods, Springer, New York, (1999). 
[2] D. Gamerman, F. H. Lopes, Markov chain Monte Carlo: stochastic simulation for Bayesian inference. CRC Press, (2006).

[3] S. Mallat: A wavelet tour of signal processing. Academic press, (1999).

[4] I. Daubechies: Ten lectures on wavelets. Society for industrial and applied mathematics, Philadelphia, (1992).

[5] A. Akansu, W. Serdijn, I. Selesnick: Wavelet Transforms in Signal Processing: A Review of Emerging Applications. Physical Communication, (2010).

[6] H. Akbari: Fast convergence of the Coiflet-Galerkin method for general elliptic BVPs. International Journal of Applied Mathematics and Computer Science. 23, 1, 17-27, (2013).

[7] H. Akbari, A.P. Engsig-Karup: Efficient physics-based model order reduction for large scale reservoir simulation, submitted.

[8] K. Aziz, A. Settari: Petroleum reservoir simulation. Chapman \& Hall, (1979).

[9] Z. Chen, G. Huan, Y. Ma: Computational methods for multi-phase flows in porous media. SIAM, (2006).

[10] D. Higdon, H. Lee, Z. Bi, A Bayesian approach to characterizing uncertainty in inverse problems using coarse and fine-scale information. IEEE Transactions on Signal Processing, Vol 50(2), (2002), Pages 389-399.

[11] M.R. Rasaei, M. Sahimi, Upscaling and simulation of waterflooding in heterogeneous reservoirs using wavelet transformations: application to the SPE-10 model. Trans. Porous Media 72, 311 (2008).

[12] M.R. Rasaei, M. Sahimi, Upscaling of the permeability by multi-scale wavelet transformations and simulation of multi-phase flows in heterogeneous porous media. Comput. Geosci. 13, 187-214, (2009).

[13] J. Fu, J. Gomez-Hernandez, A blocking Markov chain Monte Carlo method for inverse stochastic hydrogeological modeling, Math. Geosci., 41 (2), (2009), Pages 105-128.

[14] D. Oliver, L. Cunha, A. Reynolds, Markov chain Monte Carlo methods for conditioning a permeability field to pressure data, Math. Geol., 29 (1), (1997), Pages 61-91.

[15] C. Fox, G. Nicholls, Sampling conductivity images via MCMC, K. Mardia, C. Gill, R. Aykroyd (Eds.), Proceedings of the Leeds Annual Statistics Research Workshop, Leeds University Press (1997), Pages 91100.

[16] Y. Efendiev, T. Hou, W. Luo, Preconditioning Markov chain Monte Carlo simulations using coarse-scale models SIAM J. Sci. Comput., 28 (2), (2006), Pages 776-803.

[17] Y. Efendiev, A. Datta-Gupta, V. Ginting, X. Ma, B. Mallick, An efficient two-stage Markov chain Monte Carlo method for dynamic data integration, Water Resour. Res. 41 (W12423).

[18] V. Ginting, F. Pereira, A. Rahunanthan, Multi-physics Markov chain Monte Carlo methods for subsurface flows, Mathematics and Computers in Simulation, Vol. 118, (2015), Pages 224-238.

[19] A. Abedini, F. Torabi, Oil recovery performance of immiscible and miscible $\mathrm{CO}_{2}$ huff-and-puff processes. Energy \& Fuels, Vol. 28(2), (2014), Pages 774-84.

[20] B. Iraji, S. R. Shadizadeh, M. Riazi, Experimental investigation of $\mathrm{CO}_{2}$ huff and puff in a matrix-fracture system, Fuel, Vol. 158, (2015), Pages 105-112.

[21] E. Wong, Stochastic processes in information and dynamical systems, McGraw-Hill, New York, (1971).

[22] L.J. Durlofsky, Upscaling of geocellular models for reservoir simulation: a review of recent progress. 7th International Forum on Reservoir Simulation Bhl/Baden-Baden, Germany, (2003).

[23] L.J. Durlofsky, C.J. Richard, J.M. William, A nonuniform coarsening approach for the scale-up of displacement processes in heterogeneous porous media. Advances in Water Resources, 20, 5, (1997), Pages 335-347. 


\begin{tabular}{c|ccccccccc} 
& Grid & $L_{x}$ & $L_{y}$ & $\sigma_{G}^{2}$ & $N_{e v}$ & $\delta_{q}$ & $\tilde{\sigma}_{f}^{2}$ & $\tilde{\sigma}_{c}^{2}$ & $\tilde{\sigma}_{w}^{2}$ \\
\hline Case 1 & $64 \times 64$ & 0.15 & 0.15 & 4 & 30 & 0.2 & 50 & 40 & 32 \\
Case 2 & $72 \times 72$ & 0.125 & 0.125 & 5 & 40 & 0.3 & 40 & 34 & 28
\end{tabular}

Table 1: Chosen parameters in (11) and (9) for Cases 1 and 2.

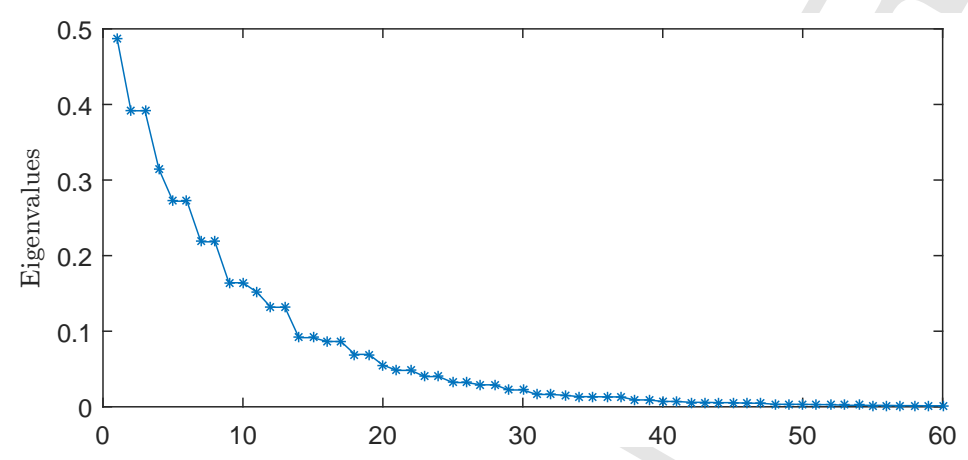

Figure 2: Decaying of eigenvalues in KL expansion (14) for Case 1.

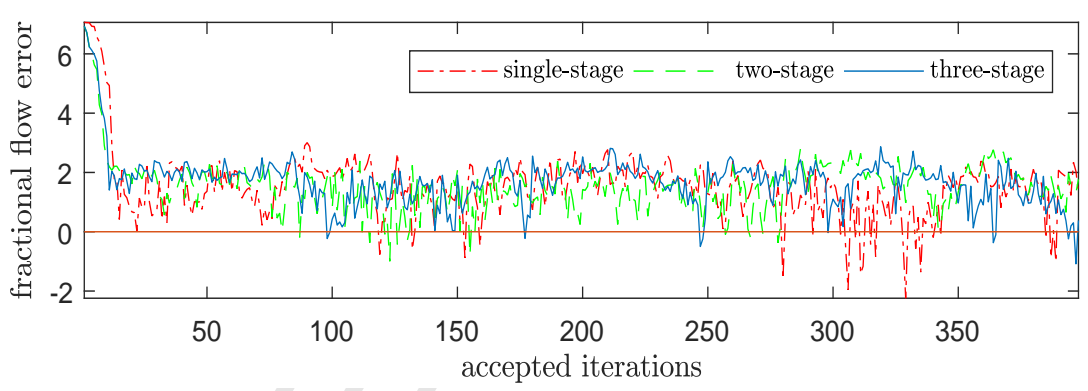

Figure 3: Shifted errors (6) for Case 1 which confirms that error levels are similar.

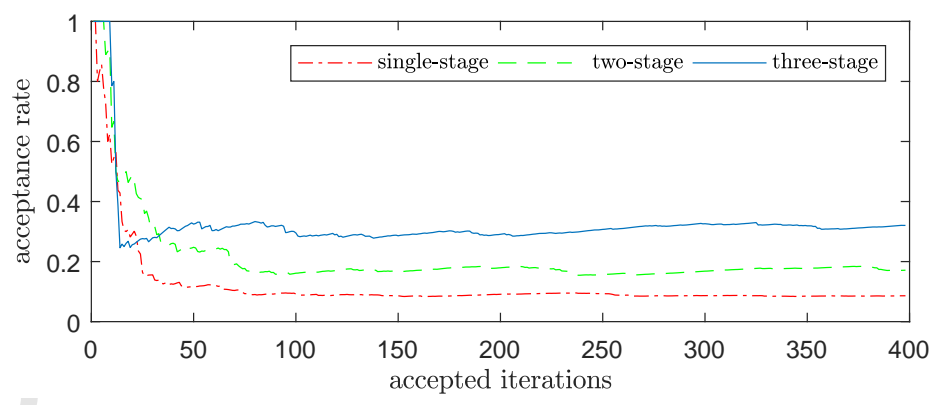

Figure 4: Acceptance rate for Case 1. 


\begin{tabular}{c|cccccc} 
MCMC Algorithm & proposals & screening wells & coarse scale sim. & forward sim. & accepted & rate \\
\hline Single-stage & 4660 & - & - & 4660 & 400 & $8.6 \%$ \\
Two-stage & 12530 & - & 12530 & 2330 & 400 & $17.2 \%$ \\
Three-stage & 11600 & 11600 & 6800 & 1250 & 400 & $32.0 \%$
\end{tabular}

Table 2: Number of evaluated proposals at each stage for Case 1.

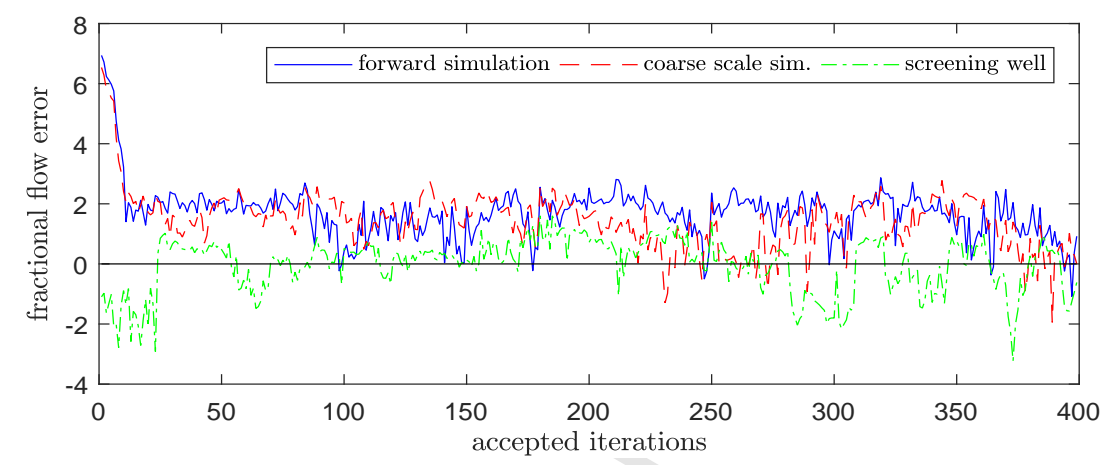

Figure 5: Shifted error (6) of three-stage MCMC for Case 1.

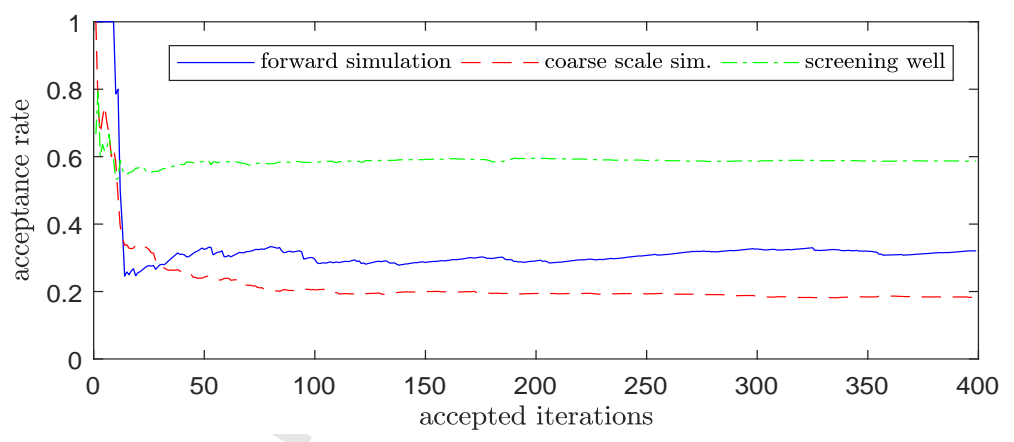

Figure 6: Acceptance rate of three stage MCMC for Case 1.

\begin{tabular}{c|cccccc} 
& proposals & screening wells & coarse scale sim. & forward sim. & accepted & rate \\
\hline single & 3950 & - & - & 3950 & 180 & $4.6 \%$ \\
two & 11050 & - & 11050 & 1266 & 180 & $14 \%$ \\
three & 13850 & 13850 & 4626 & 678 & 180 & $26.5 \%$
\end{tabular}

Table 3: Number of evaluated proposals at each stage for Case 2. 


\section{ACCEPTED MANUSCRIPI}

(1)

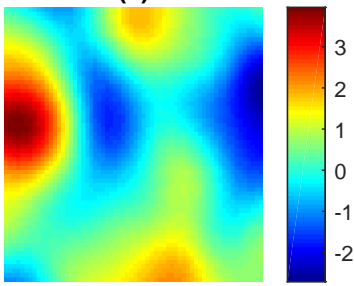

(3)

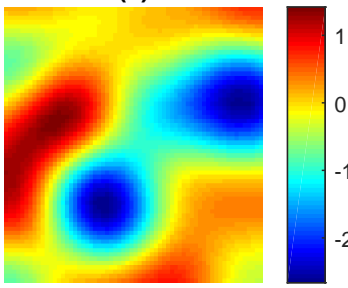

(5)

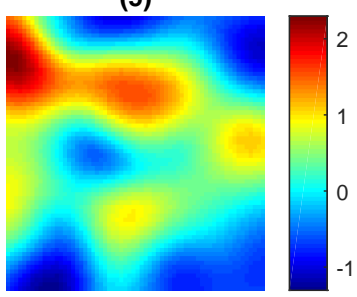

(2)

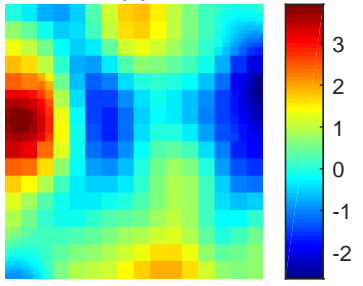

(4)

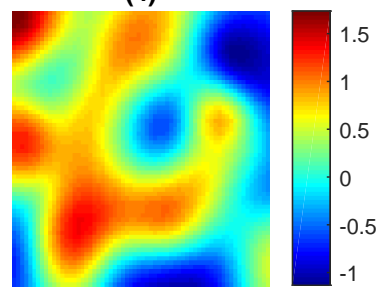

(6)

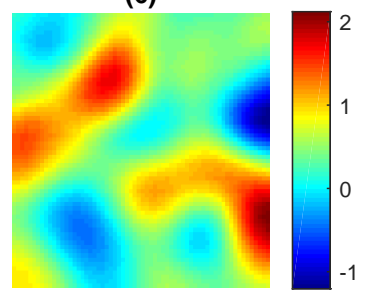

Figure 7: Permeability fields for Case 1. (1) reference (2) coarsened of reference (3) initial (4) single-stage (5) two-stage (6) three-stage.

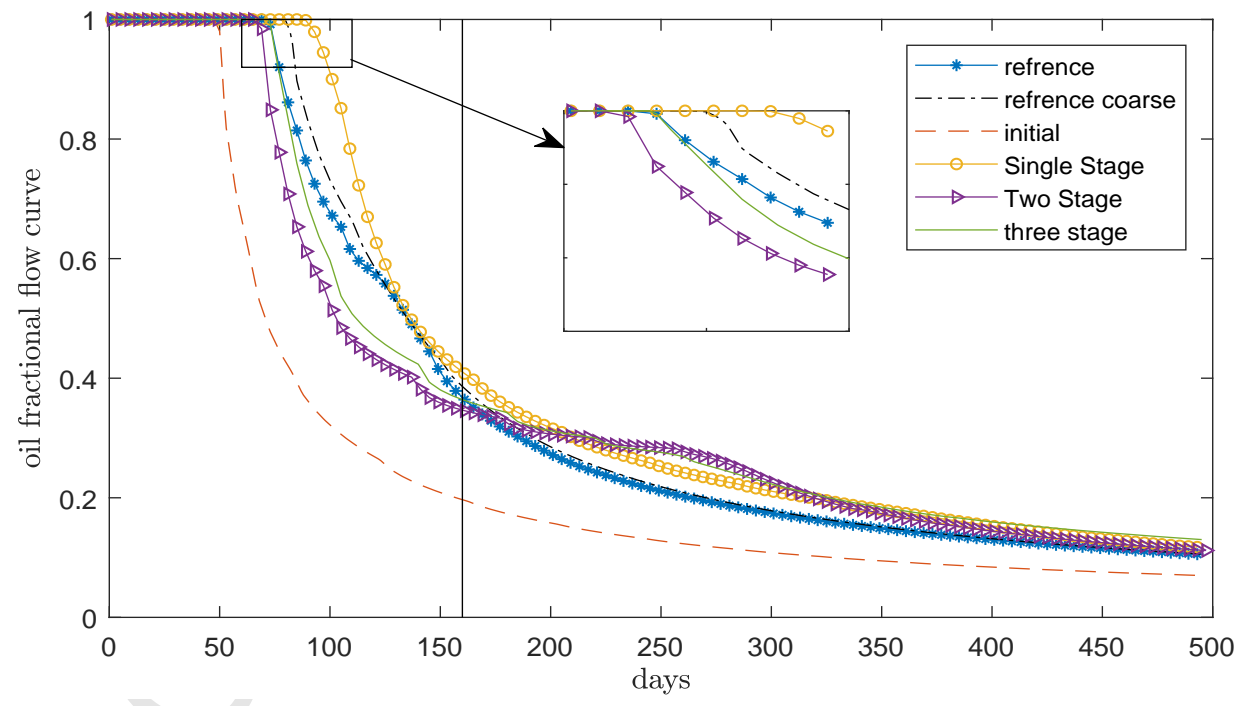

Figure 8: Oil fractional curves for predicting study for Case 1. Vertical line separates dynamic data for MCMC simulation and dynamic data for prediction. Breakthrough time is enlarged. 


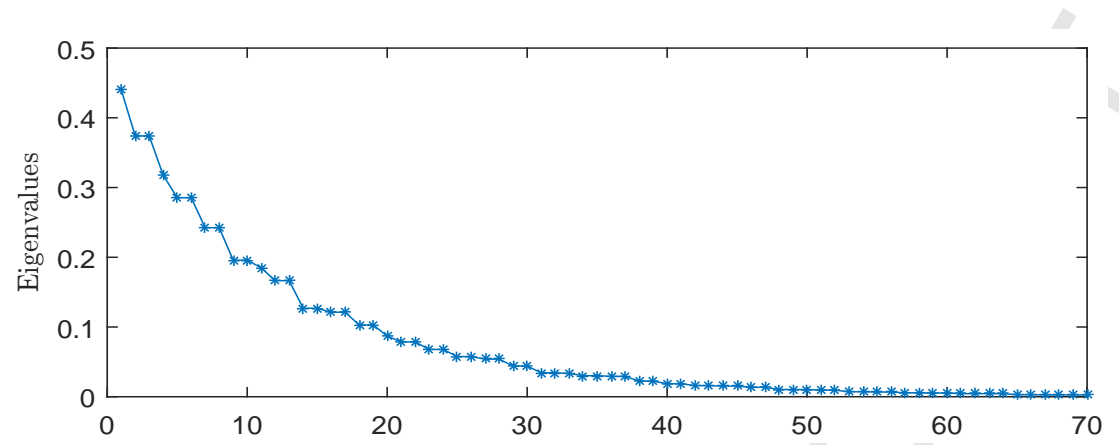

Figure 9: Decaying of eigenvalues in KL expansion (14) for Case 2.

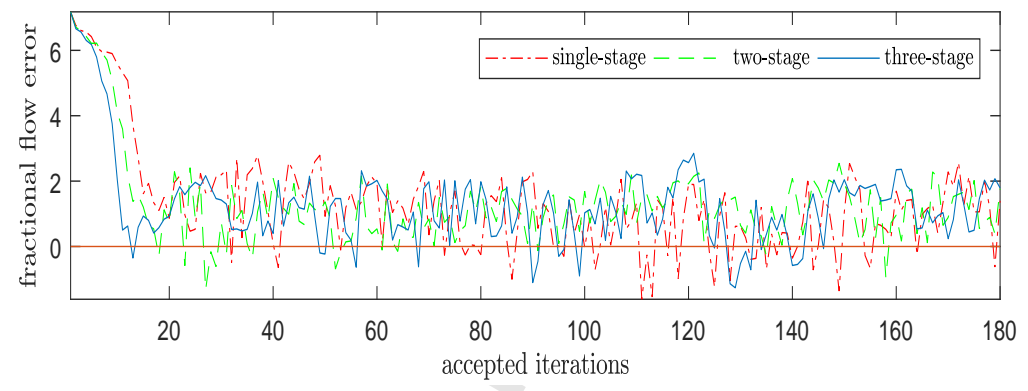

Figure 10: Shifted errors (6) for Case 2.

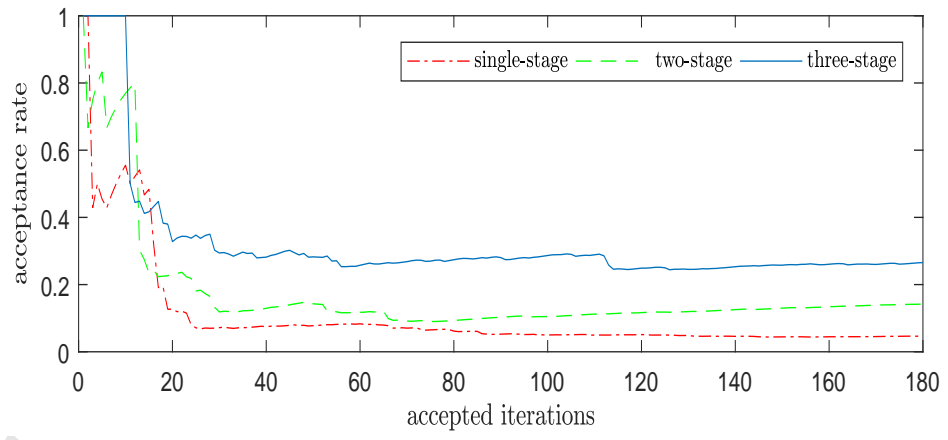

Figure 11: Acceptance rate for Case 2. 


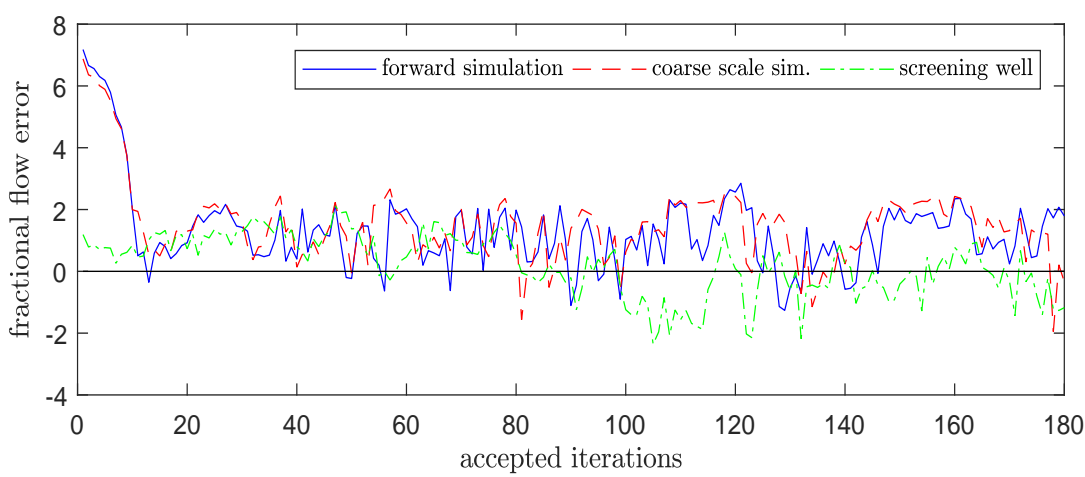

Figure 12: Shifted error (6) of three-stage MCMC for Case 2.

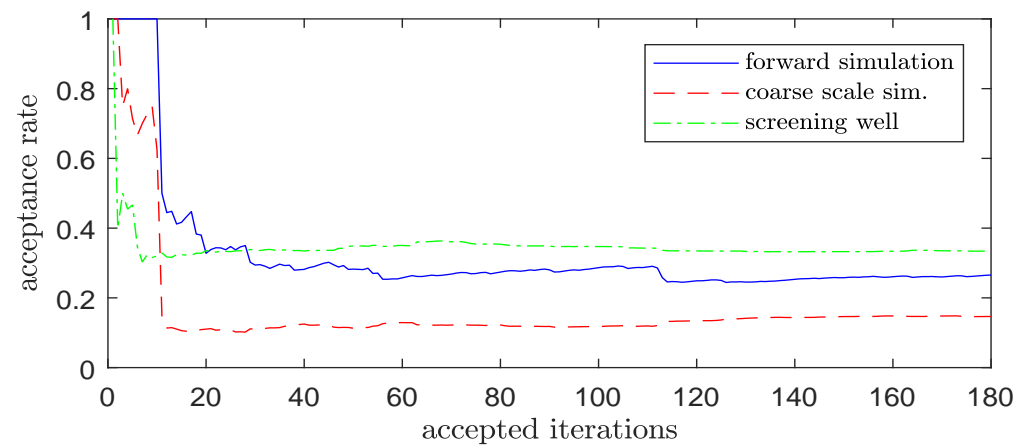

Figure 13: Acceptance rate of three stage MCMC for Case 2. 
(1)

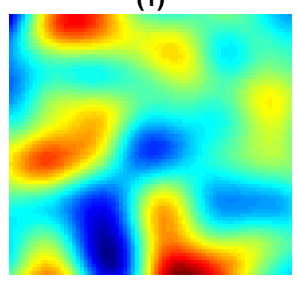

(3)

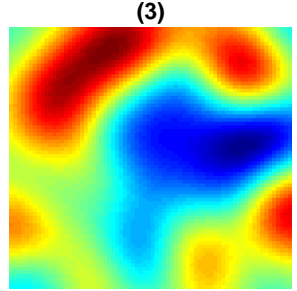

(5)

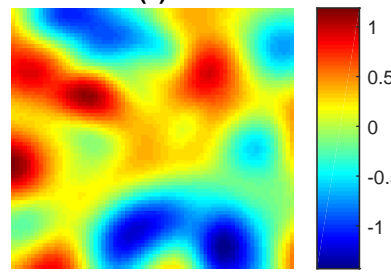

(2)
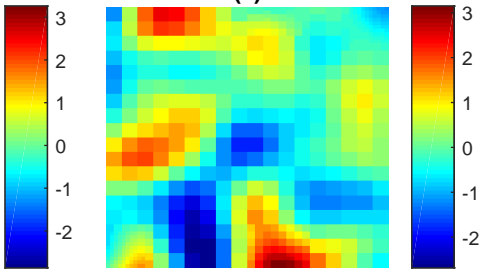

(4)

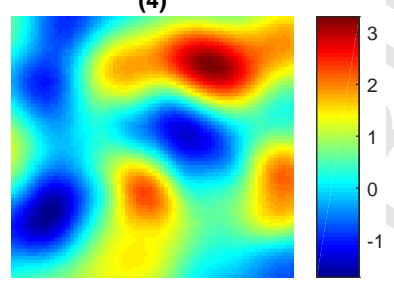

(6)

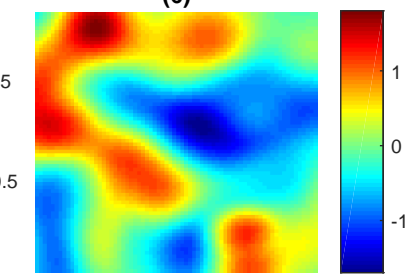

Figure 14: Permeability fields for Case 2. (1) reference (2) coarsened of reference (3) initial (4) single-stage (5) two-stage (6) three-stage.

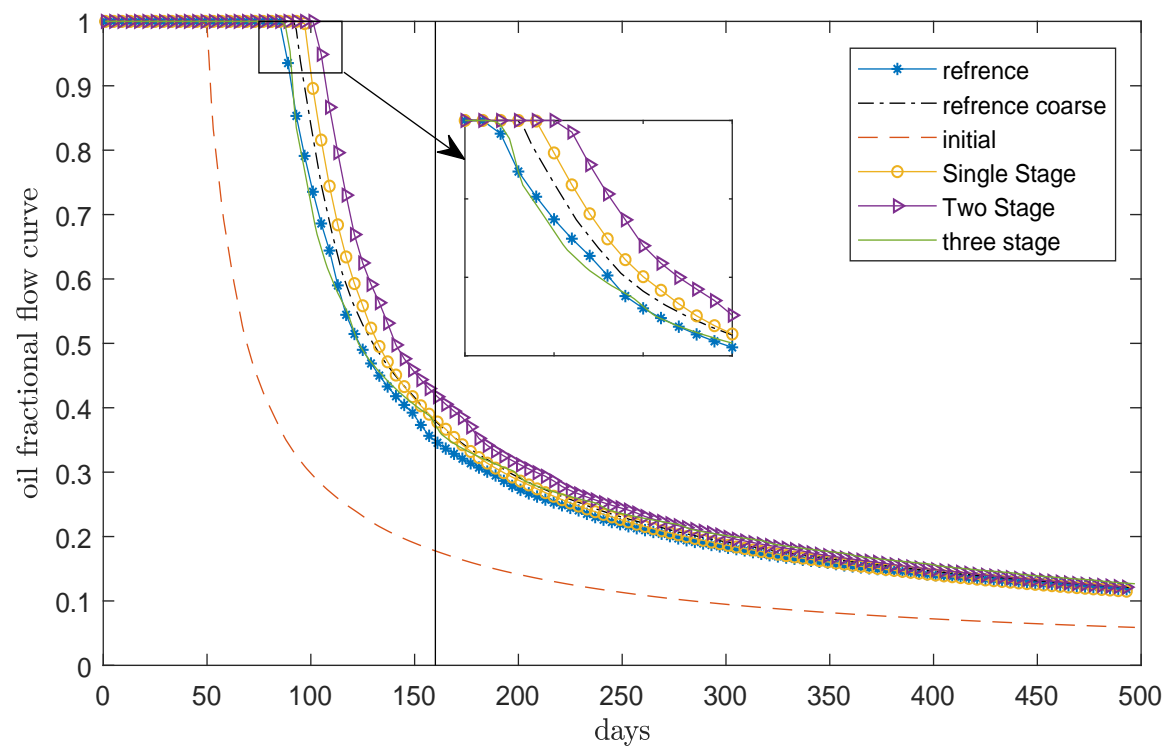

Figure 15: Oil fractional curves for predicting study for Case 2. Vertical line separates dynamic data for MCMC simulation and dynamic data for prediction. Breakthrough time is enlarged. 